
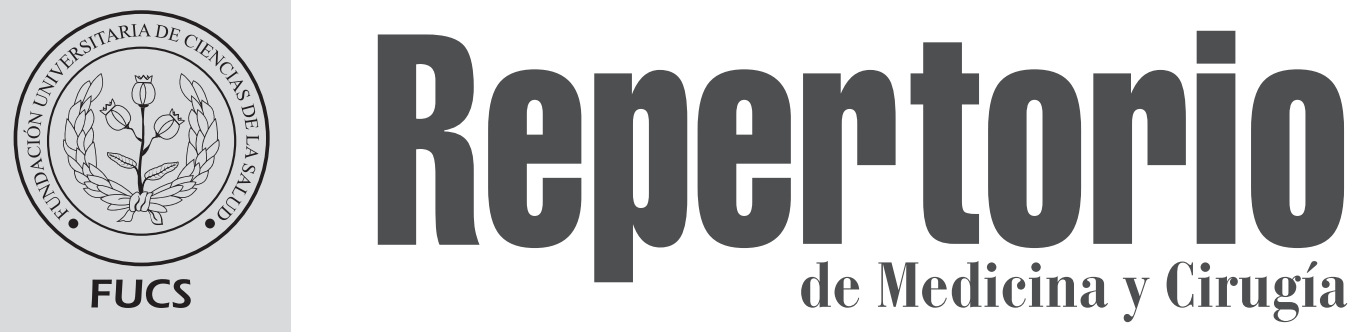

Vol. 280
№2. 2019

ISSN: 0121-7372 • ISSN electrónico: 2462-991X

Reporte de caso

\title{
Distrofia muscular de Becker con duplicación en el exón 5 del gen DMD
}

\author{
Ana Isabel Sánchez MD ${ }^{a}$ \\ Nathalie Mariño MD $^{\mathrm{b}}$ \\ Andrés Felipe Araujo MD $^{c}$ \\ Eugenia Espinosa MD $^{\mathrm{d}}$
}

\section{Becker muscular dystrophy with gene DMD exon 5 duplication}

${ }^{a}$ Genética Médica, Pontificia Universidad Javeriana, Bogotá DC, Colombia.

${ }^{b}$ Neurologia, Fundación Universitaria de Ciencias de la Salud. Bogotá DC, Colombia.

${ }^{c, d}$ Pediatría y Neurología pediátrica, Hospital Militar Central, Bogotá DC, Colombia.

${ }^{d}$ Neuropediatría, Universidad Militar Nueva Granada, Bogotá DC, Colombia.

\section{R E S U M E N}

Las distrofinopatías son un grupo de enfermedades ligadas al cromosoma X que abarcan diferentes entidades, siendo las más importantes la distrofia muscular de Duchenne (DMD) y la de Becker (DMB). Están causadas por mutaciones en el gen de la distrofina (gen DMD) localizado en el cromosoma X, locus Xp21.1. En relación con el tipo de mutaciones reportadas en el gen DMD, las delecciones y las mutaciones puntuales son las más comunes, mientras que las duplicaciones corresponden a 10-12\%. Aunque las duplicaciones que abarcan el exón 5 ya han sido reportadas en la literatura, a la fecha no existen informes de casos que establezcan una relación genotipo fenotipo clara. Presentamos el caso de un paciente con distrofia muscular de Becker con un fenotipo no tan severo, en quien se encontró una duplicación en el exón 5. Con este caso pretendemos profundizar en la relación genotipo-fenotipo de la DMB, reportando las características clínicas en relación con la duplicación del exón 5 encontrada.

Palabras clave: distrofia muscular ligada a X, distrofia muscular de Duchenne, distrofia muscular de Becker, duplicación, distrofinopatías

(C) 2018 Fundación Universitaria de Ciencias de la Salud - FUCS Este es un artículo Open Access bajo la licencia CC BY-NC-ND (http://creativecommons.org/licenses/by-nc-nd/4.0/).

\section{INFORMACIÓN DEL ARTÍCULO}

Historia del artículo:

Fecha recibido: enero 25 de 2018 Fecha aceptado: julio 30 de 2018
Autor para correspondencia. Dra. Ana Isabel Sánchez anaisanchezb@gmail.com
DOI

10.31260/RepertMedCir.v28.n1.2019.920 
The dystrophinopathies are a group of X-linked genetic disorders. The most important forms of dystrophinopathies are Duchenne muscular dystrophy (DMD) and Becker muscular dystrophy (BMD). They are caused by mutations of the dystrophin-encoding DMD gene located on the X chromosome at Xp21.1. Among the type of gene DMD mutations reported, deletions and point mutations are the most common, while duplications occur in 10-12\% of cases. Although duplications of exon 5 are already reported in the literature, to date there are no cases reported which establish a clear genotype-phenotype correlation. Here we present the case of a patient with Becker muscular dystrophy with a slightly milder phenotype, in whom exon 5 duplication was found. With this case report, we intend to highlight BMD genotype-phenotype correlation by describing BMD clinical features in relation with exon 5 duplication.

Key words: X-linked muscular dystrophy, Duchenne muscular dystrophy, Becker, muscular dystrophy, duplication, dystrophinopathies.

(C) 2018 Fundación Universitaria de Ciencias de la Salud - FUCS. This is an open access article under the CC BY-NC-ND license ( ñhttp://creativecommons.org/licenses/by-nc-nd/4.0/).

\section{IN T RODUCCIÓN}

Las distrofinopatías son un grupo de enfermedades ligadas al cromosoma $\mathrm{X}$ que abarcan diferentes entidades, siendo las más importantes la distrofia muscular de Duchenne (DMD) y la de Becker (DMB). Las distrofinopatías están causadas por mutaciones en el gen de la distrofina (gen DMD) localizado en el cromosoma X, locus Xp21.1. ${ }^{1}$ Este gen codifica para una proteína del citoesqueleto de 427 kilo daltons con diferentes isoformas, distribuidas en músculo esquelético, cerebro, células de Purkinge y músculo cardiaco. ${ }^{2}$ La variedad en el fenotipo de las dos entidades puede ser explicada por las diferentes mutaciones encontradas en el gen de la distrofina. ${ }^{3}$

La forma más severa de las distrofinopatías está dada por la $\mathrm{DMD}$, que se presenta en 1 de 3.600-6.000 recién nacidos vivos masculinos, representando el $80-85 \%$ de los casos de distrofinopatías. ${ }^{4}$ La DMB tiene una prevalencia de 1 en 18.000 recién nacidos vivos. ${ }^{5}$

Clínicamente la DMD se caracteriza por debilidad muscular progresiva de aparición temprana (2-3 años), que puede estar acompañada de antecedente de retraso motor del desarrollo. La deambulación se compromete alrededor de los 13 años y aparece la cardiomiopatía dilatada. Los pacientes fallecen entre la primera y segunda década de la vida a consecuencia de falla respiratoria por debilidad de músculos intercostales y diafragma $(75 \%)$, así como por falla cardiaca $(20 \%) .{ }^{4,6} \mathrm{La}$ DMB presenta un fenotipo un poco más leve, usualmente los pacientes afectados conservan la deambulación inclusive hasta los 16 años y pueden vivir hasta los 65 años. ${ }^{7}$

El diagnóstico en DMD/DMB incluye la sospecha clínica y la historia familiar positiva de distrofinopatías, en combinación con ayudas diagnósticas como CK en suero que se encuentra muy elevada, patrón electromiográfico con alteración intrínseca de la fibra muscular sin evidente alteración de la conducción nerviosa y biopsia muscular en algunos casos, con determinación mediante inmunohistoquímica o Western Blot de la proteína distrofina. ${ }^{8}$ El diagnóstico molecular es posible en nuestro medio. ${ }^{8}$

Las metodologías actuales para detectar la presencia de mutaciones en el gen DMD incluyen múltiplex PCR, Souther blotting, MLPA (multiplex ligation-dependent probe amplification) y secuenciación de DNA mediante NGS o Sanger. ${ }^{9-13}$

Presentamos el caso de un paciente afectado con distrofia muscular de Becker con un fenotipo no tan severo, en quien se encontró una duplicación en el exón 5. Con este caso pretendemos ahondar en la relación genotipo-fenotipo de la $\mathrm{DMB}$, reportando las características clínicas en relación con la duplicación del exón 5 encontrada.

\section{REPORTE DEL CASO}

Escolar masculino 15 años de edad producto del primer embarazo de padres no consanguíneos. La gestación fue controlada sin presentar patologías maternas y las ecografías prenatales fueron normales. Recién nacido a término, peso al nacer: $3600 \mathrm{~g}$, talla de $50 \mathrm{cms}$, sin patologías neonatales.

En relación con su desarrollo psicomotor, inició sostén cefálico a los 3 meses, sedestación a los 8 meses y marcha a los 12 meses. En el lenguaje presentó dislalia a los dos años, que mejoró con terapias de lenguaje.

A los 4 años comienza a presentar dificultad para la marcha con caídas frecuentes, debilidad y fatigabilidad muscular que progresó hasta la dificultad para subir o bajar escaleras, para saltar o para levantarse del suelo por sus propios medios, requiriendo apoyos adicionales. 
En la primera valoración por neurología pediátrica a los 10 años se encontró al examen físico marcha independiente con patrón miopático, signo de Gowers positivo, hipertrofia bilateral de gastrocnemios, escápula alada bilateral y fuerza muscular conservada en miembros superiores y disminuida 4/5 en músculos proximales de miembros inferiores.

Como parte del enfoque diagnóstico de distrofia muscular se solicitaron CPK con resultado en 14,175 U/L y electromiografía que reportó un estudio anormal con compromiso de la fibra muscular intrínseca, estadío subagudo en 4 extremidades (patrón miopático vs distrofia muscular), sin alteración en velocidades de neuroconducción. Además se documentó mioglobina elevada en suero 288 y transaminasas ligeramente altas (AST 180, ALT 200). Serología no reactiva, estudios reumatológicos incluyendo ANAs, ANCAs normales y RMN cerebral y ecocardiograma normales. Con base en la clínica del paciente y en los paraclínicos que evidenciaron elevación significativa de la CPK, se sospechó distrofia muscular de Duchenne vs Becker. El paciente se siguió clínicamente con controles por neurología pediátrica, encontrándose valores de CPK que oscilaban entre 10,000 y 16,000 U/L. Se inició manejo terapéutico con prednisolona a $0.8 \mathrm{mg} / \mathrm{k} /$ día, hasta incrementar dosis de $1 \mathrm{mg} / \mathrm{k} /$ día cuando alcanzó $40 \mathrm{k}$ de peso.

En la actualidad el paciente tiene 16 años, se encuentra en tratamiento con prednisolona $40 \mathrm{mg} /$ día, ha persistido con transaminitis, por lo que se realizó biopsia de hígado que fue normal. Al examen físico se detecta arreflexia de RMT rotulianos bilaterales, aumento en la hipertrofia de gastrocnemios, fuerza de $4 / 5$ en 4 extremidades, marcha en estepaje con pie caído y tendencia al equino. Además presencia de retracciones isquiotibiales y aquilianas. El ecocardiograma de control a esta edad evidenció leve hipoquinesia de pared posterior ventricular con FE de ventrículo izquierdo de $51 \%$.

El paciente se encuentra en terapias físicas desde el año 2014, con el objetivo terapéutico de preservar en el músculo la función y evitar la disminución del trofismo muscular. Valorado por genética médica, se solicitó MLPA para el gen de la distrofina (DMD), encontrándose una duplicación en el exón 5 (c.265_357dup) y confirmando el diagnóstico molecular de distrofinopatía. Por el cuadro clínico lentamente progresivo, se consideró el diagnóstico molecular compatible con distrofia muscular de Becker.

\section{DISCUSIÓN Y CONCLUSIONES}

Presentamos un paciente masculino de 16 años, caso único en la familia, con cuadro clínico compatible con DMB y confirmación molecular mediante análisis de rearreglos con MLPA, en el que se encontró duplicación del exón 5 del gen DMD. Este es uno de los genes más grandes que existen, conteniendo cerca de 79 exones. La severidad clínica de la DMD depende de qué tanto esté alterado el marco de lectura secundario a la mutación encontrada. Deleciones por fuera del marco de lectura, como norma generan ausencia completa de distrofina o niveles muy bajos dando lugar a fenotipos severos ${ }^{5}$, mientras que deleciones que mantienen el marco de lectura, producen una proteína de bajo peso molecular, poco funcional, que resulta en el fenotipo de DMB ${ }^{14,15}$ Esta regla del marco de lectura, se encuentra en casi el $90 \%$ de los casos. ${ }^{16}$

En cuanto al tipo de mutaciones reportadas en el gen DMD, las deleciones se presentan en el 60-65\% de los casos de DMD o DMB, mientras que las puntuales (pequeñas deleciones intragénicas, inserciones y cambios en secuencia) ocurren 25 a $30 \%$ de los pacientes. $^{3}$ Las mutaciones de tipo non-sense se ven en $13 \%$ de los afectados con $\mathrm{DMD}^{3}$ y las duplicaciones del gen se han encontrado en $10-12 \%$ de los casos. ${ }^{3}$ La mayoría de las deleciones ocurren en los exones 45 a 53, por lo cual a esta región génica se le conoce como "hot spot exons", mientras que las duplicaciones ocurren por lo regular en los exones 2 a $20 .{ }^{17}$

Aunque las duplicaciones que abarcan el exón 5 han sido reportadas en la literatura ${ }^{12,13}$, hasta la fecha no existen casos que establezcan una relación genotipo fenotipo clara cuando este único exón se encuentra duplicado, como es el caso de nuestro paciente.

A nivel mundial se han hecho varios estudios para caracterizar las mutaciones y establecer relaciones genotipo fenotipo en grandes cohortes de pacientes. ${ }^{18-23}$ Entre los pacientes afectados con distrofia muscular de Duchenne/Becker en quienes se encuentran duplicaciones en el gen, es difícil hacer suposiciones con respecto a si el marco de lectura está alterado o no, y por ende a predecir un fenotipo definitivo. ${ }^{20}$ Aunque las duplicaciones en tándem son comunes ${ }^{22}$, están descritos otros tipos de duplicaciones que no están en tándem ${ }^{23}$, por eso para la determinación de la orientación del fragmento duplicado es necesario realizar análisis directo en el RNA mensajero. ${ }^{20}$ En muchas cohortes en las que se estudia el genotipo de este tipo de distrofias se reporta que la duplicación más común es la duplicación del exón $2 .^{20,23}$

La c.265_357dup se trata de una mutación localizada en el dominio de unión a la actina, en el codón 89 del exón 5 del gen. Con base en el fenotipo del paciente suponemos que no modifica el marco de lectura, permitiendo la presencia de proteína en la fibra muscular y teniendo como consecuencia un cuadro clínico leve, lentamente progresivo e inclusive con poca afectación cardiaca.

La transaminitis persistente hallada en el paciente se explica en la literatura mundial por la presencia de enzimas hepáticas alteradas en casos con distrofia muscular, que puede ser secundaria a la degradación de la fibra muscular más que a alguna patología hepática e inclusive se sugiere que estos enfermos no deberían ser investigados para afecciones del hígado. ${ }^{24}$ Es más, en ausencia de patología hepática las transaminasas elevadas 
pueden ser un signo temprano de distrofia muscular oculta y por ende deben investigarse niveles de CPK en busca de evidencia de distrofia muscular. ${ }^{24}$

Es de gran importancia conocer que a nivel mundial se han hecho grandes esfuerzos para encontrar tratamientos oportunos y efectivos para las distrofias musculares. Se vienen realizando múltiples estudios de terapia génica, algunos inclusive ya en fase clínica, que parecen ser bastante prometedores, incluyendo CRISPR-CAS9 y oligonucleótidos antisentido entre otros. ${ }^{25-28}$ Es más, la generación de la molécula de Ataluren, que actúa como un codón de stop, ha sido revolucionaria en el manejo de $\mathrm{DMD} / \mathrm{DMB}$ secundarias a mutaciones puntuales de tipo nonsense, siempre y cuando el paciente todavía conserve la deambulación. ${ }^{29}$

Hasta la fecha el manejo estándar para las distrofias musculares de Duchenne/Becker continúa siendo la terapia anti inflamatoria con esteroides orales, que tiene como fin la prevención de un mayor desgaste de la fibra muscular ${ }^{30}$, existiendo numerosas estrategias para prevenir los efectos secundarios de estos medicamentos. $^{31}$ El paciente aquí reportado se encuentra en terapia con esteroides orales, la cual ha favorecido una progresión lenta de la enfermedad.

Este reporte de caso pone en evidencia un paciente de 16 años con una evolución clínica bastante favorable de la historia natural de la enfermedad. La confirmación molecular del diagnóstico fue posible, lo que nos permite establecer una relación genotipo fenotipo específica, en la que se puede concluir que duplicaciones en el gen, específicamente en el exón 5, parecieran permitir la presencia de distrofina, que aunque disfuncional, puede ser suficiente para que el cuadro clínico sea atenuado y la progresión lenta.

\section{CONFLICTO DE INTERÉS}

Los autores declaran no tener ningún conflicto de intereses.

\section{REFEREN CIAS}

1. Blake DJ, Weir A, Newey SE, Davies KE. Function and genetics of dystrophin and dystrophin-related proteins in muscle. Physiological reviews. 2002;82(2):291-329. doi: 10.1152/ physrev.00028.2001.

2. Muntoni F, Melis MA, Ganau A, Dubowitz V. Transcription of the dystrophin gene in normal tissues and in skeletal muscle of a family with X-linked dilated cardiomyopathy. American journal of human genetics. 1995;56(1):151-7.

3. Muntoni F, Torelli S, Ferlini A. Dystrophin and mutations: one gene, several proteins, multiple phenotypes. The Lancet Neurology. 2003;2(12):731-40.
4. Bushby K, Finkel R, Birnkrant DJ, Case LE, Clemens PR, Cripe L, et al. Diagnosis and management of Duchenne muscular dystrophy, part 1: diagnosis, and pharmacological and psychosocial management. The Lancet Neurology. 2010;9(1):77-93. doi: 10.1016/S1474-4422(09)70271-6.

5. Hoffman EP, Fischbeck KH, Brown RH, Johnson M, Medori $\mathrm{R}$, Loike JD, et al. Characterization of dystrophin in musclebiopsy specimens from patients with Duchenne's or Becker's muscular dystrophy. The New England journal of medicine. 1988;318(21):1363-8. doi: 10.1056/NEJM198805263182104

6. Essex C, Roper H. Lesson of the week: late diagnosis of Duchenne's muscular dystrophy presenting as global developmental delay. BMJ. 2001;323(7303):37-8.

7. Bushby KM, Gardner-Medwin D, Nicholson LV, Johnson MA, Haggerty ID, Cleghorn NJ, et al. The clinical, genetic and dystrophin characteristics of Becker muscular dystrophy. II. Correlation of phenotype with genetic and protein abnormalities. Journal of neurology. 1993;240(2):105-12.

8. Ministerio de Salud y Protección Social, Departamento Administrativo de Ciencia Tecnología e Innovación - Colciencias. Guía de práctica clínica, para la detección temprana, atención integral, seguimiento y rehabilitación de pacientes con diagnóstico de distrofia muscular. Guía No. 37. Bogotá: Ministerio de Salud y Protección Social; 2015. p. 694.

9. Hegde MR, Chin EL, Mulle JG, Okou DT, Warren ST, Zwick ME. Microarray-based mutation detection in the dystrophin gene. Human mutation. 2008;29(9):1091-9. doi: 10.1002/humu.20831.

10. Stockley TL, Akber S, Bulgin N, Ray PN. Strategy for comprehensive molecular testing for Duchenne and Becker muscular dystrophies. Genetic testing. 2006;10(4):229-43. doi: 10.1089/gte.2006.10.229.

11. Gatta V, Scarciolla O, Gaspari AR, Palka C, De Angelis MV, Di Muzio A, et al. Identification of deletions and duplications of the DMD gene in affected males and carrier females by multiple ligation probe amplification (MLPA). Human genetics. 2005;117(1):92-8. doi: 10.1007/s00439-005-1270-7.

12. Janssen B, Hartmann C, Scholz V, Jauch A, Zschocke J. MLPA analysis for the detection of deletions, duplications and complex rearrangements in the dystrophin gene: potential and pitfalls. Neurogenetics. 2005;6(1):29-35. doi: 10.1007/s10048-004-0204-1.

13. Hamed SA, Hoffman EP. Automated sequence screening of the entire dystrophin cDNA in Duchenne dystrophy: point mutation detection. American journal of medical genetics Part B, Neuropsychiatric genetics : the official publication of the International Society of Psychiatric Genetics. 2006;141B(1):44-50. doi: 10.1002/ajmg.b.30234.

14. Nicholson LV, Johnson MA, Gardner-Medwin D, Bhattacharya S, Harris JB. Heterogeneity of dystrophin expression in patients with Duchenne and Becker muscular dystrophy. Acta neuropathologica. 1990;80(3):239-50.

15. Monaco AP, Bertelson CJ, Liechti-Gallati S, Moser H, Kunkel LM. An explanation for the phenotypic differences between patients bearing partial deletions of the DMD locus. Genomics. 1988;2(1):90-5. 
16. Aartsma-Rus A, Van Deutekom JC, Fokkema IF, Van Ommen GJ, Den Dunnen JT. Entries in the Leiden Duchenne muscular dystrophy mutation database: an overview of mutation types and paradoxical cases that confirm the reading-frame rule. Muscle \& nerve. 2006;34(2):135-44. doi: 10.1002/mus.20586.

17. Vengalil S, Preethish-Kumar V, Polavarapu K, Mahadevappa M, Sekar D, Purushottam M, et al. Duchenne Muscular Dystrophy and Becker Muscular Dystrophy Confirmed by Multiplex Ligation-Dependent Probe Amplification: Genotype-Phenotype Correlation in a Large Cohort. J Clin Neurol. 2017;13(1):91-7. doi: 10.3988/jcn.2017.13.1.91.

18. Yang J, Li SY, Li YQ, Cao JQ, Feng SW, Wang YY, et al. MLPAbased genotype-phenotype analysis in 1053 Chinese patients with DMD/BMD. BMC medical genetics. 2013;14:29. doi: 10.1186/14712350-14-29.

19. Guo R, Zhu G, Zhu H, Ma R, Peng Y, Liang D, et al. DMD mutation spectrum analysis in 613 Chinese patients with dystrophinopathy. Journal of human genetics. 2015;60(8):435-42. doi: 10.1038/ jhg.2015.43.

20. Flanigan KM, Dunn DM, von Niederhausern A, Soltanzadeh P, Gappmaier E, Howard MT, et al. Mutational spectrum of DMD mutations in dystrophinopathy patients: application of modern diagnostic techniques to a large cohort. Human mutation. 2009;30(12):1657-66. doi: 10.1002/humu.21114.

21. Juan-Mateu J, Gonzalez-Quereda L, Rodriguez MJ, Baena M, Verdura E, Nascimento A, et al. DMD Mutations in 576 Dystrophinopathy Families: A Step Forward in GenotypePhenotype Correlations. PloS one. 2015;10(8):e0135189. doi: 10.1371/journal.pone.0135189.

22. Hu XY, Ray PN, Worton RG. Mechanisms of tandem duplication in the Duchenne muscular dystrophy gene include both homologous and nonhomologous intrachromosomal recombination. The EMBO journal. 1991;10(9):2471-7.

23. White SJ, Aartsma-Rus A, Flanigan KM, Weiss RB, Kneppers AL,
Lalic T, et al. Duplications in the DMD gene. Human mutation. 2006;27(9):938-45. doi: 10.1002/humu.20367.

24. Tay SK, Ong HT, Low PS. Transaminitis in Duchenne's muscular dystrophy. Annals of the Academy of Medicine, Singapore. 2000;29(6):719-22.

25. Rinaldi C, Wood MJA. Antisense oligonucleotides: the next frontier for treatment of neurological disorders. Nature reviews Neurology. 2018;14(1):9-21. doi: 10.1038/nrneurol.2017.148.

26. Nelson CE, Robinson-Hamm JN, Gersbach CA. Genome engineering: a new approach to gene therapy for neuromuscular disorders. Nature reviews Neurology. 2017;13(11):647-61. doi: 10.1038/nrneurol.2017.126.

27. Lattanzi A, Duguez S, Moiani A, Izmiryan A, Barbon E, Martin S, et al. Correction of the Exon 2 Duplication in DMD Myoblasts by a Single CRISPR/Cas9 System. Molecular therapy Nucleic acids. 2017;7:11-9. doi: 10.1016/j.omtn.2017.02.004.

28. Mah JK. An Overview of Recent Therapeutics Advances for Duchenne Muscular Dystrophy. Methods Mol Biol. 2018;1687:317. doi: 10.1007/978-1-4939-7374-3_1.

29. McDonald CM, Campbell C, Torricelli RE, Finkel RS, Flanigan $\mathrm{KM}$, Goemans $\mathrm{N}$, et al. Ataluren in patients with nonsense mutation Duchenne muscular dystrophy (ACT DMD): a multicentre, randomised, double-blind, placebo-controlled, phase 3 trial. Lancet. 2017;390(10101):1489-98. doi: 10.1016/S01406736(17)31611-2.

30. Yilmaz O, Karaduman A, Topaloglu H. Prednisolone therapy in Duchenne muscular dystrophy prolongs ambulation and prevents scoliosis. European journal of neurology. 2004;11(8):541-4. doi: 10.1111/j.1468-1331.2004.00866.x.

31. Bell JM, Shields MD, Watters J, Hamilton A, Beringer T, Elliott $\mathrm{M}$, et al. Interventions to prevent and treat corticosteroid-induced osteoporosis and prevent osteoporotic fractures in Duchenne muscular dystrophy. The Cochrane database of systematic reviews. 2017;1:CD010899. doi: 10.1002/14651858.CD010899.pub2. 